\title{
Antibacterial Activity Of Shin'iseihaito (Xinyiqingfeitang) And its Components Against Methicillin-Resistant Staphylococcus aureus
}

\author{
Toru Konishi ${ }^{1}$, Masaaki Minami ${ }^{2}$, Toshiaki Makino ${ }^{{ }^{*}}$
}

\section{Toru Konishi ${ }^{1}$, Masaaki Minami², Toshiaki Makino $^{*}$}

\section{'Department of Pharmacognosy, Graduate School of Pharmaceutical Sciences, Nagoya City University, 3-1 Tanabe-dori, Mizuho-ku, Nagoya 467-8603, JAPAN. \\ 2Department of Bacteriology, Graduate School of Medical Sciences, Nagoya City University, 1 Kawasumi, Mizuho-cho, Mizuho-ku, Nagoya 467-8601, JAPAN.}

\section{Correspondence}

Toshiaki Makino,

Department of Pharmacognosy, Affiliation: Graduate School of Nagoya City University, Address: 3-1 Tanabe-dori, Mizuho-ku, Nagoya 467-8603, JAPAN.

Tel.: +81528363416

E-mail: makino@phar.nagoya-cu.ac.jp

History

- Submission Date: 11-01-17;

- Review completed: 02-03-17.

- Accepted Date: 13-03-17

DOI : 10.5530/pj.2017.3.53

Article Available online

http://www.phcogj.com/v9/i3

Copyright

(C) 2017 Phoog.Net. This is an openaccess article distributed under the terms of the Creative Commons Attribution 4.0 International license.

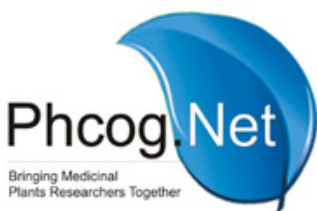

\begin{abstract}
Objective: Shin'iseihaito (xinyiqingfeitang in Chinese, SSHT), a formula in traditional Japanese Kampo medicine and Chinese medicine comprising nine crude drugs, Gypsum, Ophiopogon Tuber, Scutellaria Root (SR, root of Scutellaria baicalensis), Gardenia Fruit, Anemarrhena Rhizome, Lilium Bulb, Magnolia Flower, Loquat Leaf, and Cimicifuga Rhizome (CR, rhizome of Cimicifuga heracleifolia), is commonly used to treat sinusitis associated with purulent nasal discharge and reddish nasal mucosa. We evaluated anti-bacterial activity of SSHT extract on methicillin-resistant Staphylococcus aureus (MRSA), one cause of bacterial sinusitis. Materials and Methods: Sterile paper disks impregnated with SSHT extract, the combination of crude drugs composing SSHT according to the traditional pharmacological theory, or each component were placed on Mueller-Hinton agar plates inoculated with several strains of MRSA isolated from the patients. The diameter of inhibitory zone was measured after 18-24 h incubation. Results: SSHT extract showed antibacterial activity against 128/190 (66.8\%) MRSA clinical isolates. The effect of the extract of SSHT without heat-clearing drugs (SSHT-HC) or without exterior-releasing drugs (SSHT-ER) were significantly lower than that of SSHT extract. Each water extract of SR, Loquat Leaf, Magnolia Flower and CR showed significant anti-MRSA activity, and SR extract exhibited the largest inhibitory zone. Conclusions: SSHT has antibacterial activity against MRSA clinical isolates, and SR mainly contributes to the antibacterial activity of SSHT against MRSA clinical isolates.

Key words: Shin'iseihaito, Sinusitis, Methicillin-resistant Staphylococcus aureus (MRSA), Antibacterial activity, Scutellaria baicalensis.
\end{abstract}

\section{INTRODUCTION}

Sinusitis is the commonest disease, and decreases quality-of-life as a result of symptoms such as nasal discharge, nasal congestion, hyposmia, facial pain or pressure, headache, and malaise. ${ }^{1-3}$ Bacterial sinusitis can cause rare but severe orbital and intracranial complications such as preseptal or orbital cellulitis, subperiosteal or orbital abscess, cavernous sinus thrombosis, subdural or epidural empyema, cerebritis, brain abscess and meningitis. ${ }^{4}$

One of pathogens for bacterial sinusitis is methicillinresistant Staphylococcus aureus (MRSA), ${ }^{5,6}$ and recently, the rate of recovery of MRSA is increasing in patients with sinusitis. ${ }^{7}$ MRSA is resistant to $\beta$-lactams, and often resistant to other antibacterial agent such as macrolides, tetracyclines, aminoglycosides, and fluoroquinolones. ${ }^{8}$ Vancomycin is prescribed for the treatment of MRSA infections, ${ }^{8}$ however, the emergence of vancomycin-resistant Staphylococcus aureus was reported. ${ }^{9,10}$ Therefore, the treatment other than antibiotic therapy is demanded.

Shin'iseihaito (xinyiqingfeitang in Chinese, SSHT) is a formula in traditional Japanese Kampo medicine and Chiniese medicine to treat sinusitis associated with lung-heat syndrome, which is characterized by the symptoms of purulent nasal discharge, reddish nasal mucosa, and so on. ${ }^{11}$ SSHT composes nine crude drugs, Gypsum (G), Scutellaria Root (SR), Gardenia Fruit (GF), Anemarrhena Rhizome (AR), Ophiopogon Tuber (OT), Lilium Bulb (LB), Loquat Leaf (LL), Magnolia Flower (MF), and Cimicifuga Rhizome (CR). ${ }^{11}$ In our previous study, we showed the anti-infectious effect of SSHT extract against pneumococcus-infected mice, ${ }^{12}$ and the antibacterial activity of SSHT extract against Streptococcus pneumoniae in vitro $^{13}$. However, the antibacterial activity of SSHT against MRSA has not been investigated.

In this study, we evaluated the antibacterial activities of SSHT hot water extract and its constituents against MRSA in vitro, and discussed the contribution of each crude drugs composing SSHT according to the traditional medicinal theory.

\section{MATERIALS AND METHODS}

\section{Preparation of SSHT}

The materials of SSHT were the same lots of crude drugs in our previous study. ${ }^{13}$ Each crude drug (5 g), the mixture of crude drugs constituting SSHT (total $28 \mathrm{~g}$, Table 1) or each SSHT prescription (Table 2) were boiled in 20-times weight of distilled water 
for $30 \mathrm{~min}$ and filtrated, lyophilized, and the dried powdered extracts of SSHT, each crude drug or each SSHT prescription were weighed. The yields of the extracts to the original crude drugs were shown in Table 1 and Table 2. The powdered extracts were suspended in distilled water and stored in $-20^{\circ} \mathrm{C}$ until use. The fingerprint chromatogram of SSHT extract was almost similar to the previous study. ${ }^{13}$

\section{Bacterial strains and disk diffusion method}

One hundred ninety methicillin-resistant Staphylococcus aureus (MRSA) clinical isolates were obtained from 190 different patients admitted to Daido Hospitals, Nagoya, Japan. All Staphylococcus isolates were identified by standard conventional biochemical methods or the VITEK2 system (bioMérieux, Durham, NC, USA). Minimal inhibitory concentrations (MICs) were determined at clinical laboratory in Daido Hospital using broth micro dilution methodology with the VITEK2 system. Evaluation of susceptibilities were calculated based on Clinical Laboratory Standard Institute (CLSI) break point. Antibacterial activity was evaluated by the disk diffusion method, as described previously. ${ }^{13}$ Briefly, sterile paper disks $(12 \mathrm{~mm}$ ) were impregnated with samples, and dried up at room temperature. The colonies of MRSA which cultured overnight on LB agar (Sigma-Aldrich, St. Louis, MO, USA) at $37^{\circ} \mathrm{C}$, were collected into sterile saline and adjusted to $0.5 \mathrm{McFarland}$ using sterile saline, and inoculated on Mueller-Hinton agar (Becton Dickinson, Franklin Lakes, NJ, USA). The paper disks were placed on the plates, and were incubated at $37^{\circ} \mathrm{C}$ for $18-24 \mathrm{~h}$. Then, the diameter of inhibitory zone was measured.

\section{Statistical Analysis}

All statistical analyses were performed using the PASW Statistics version 18 (SPSS, IBM, Armonk, NY, USA). The statistical analysis was conducted using a one-way analysis of variance (ANOVA) followed by Bonferroni/ Dunnett's multiple $t$-test for the differences among multiple groups. $P$-values less than 0.05 were considered statistically significant.

\section{RESULTS AND DISCUSSION}

Antibacterial activity screening test of SSHT extract against 190 MRSA clinical isolates was evaluated by using disk diffusion method. SSHT extract $(7.5 \mathrm{mg} /$ disk $)$ exhibited antibacterial activity against 127 MRSA clinical isolates (66.8\%). Among them, we selected 3 MRSA clinical

Table 1: Compositions of shin'iseihaito (SSHT).

\begin{tabular}{ccc}
\hline Name of crude drug $^{\mathrm{a}}$ & Weight $^{\mathrm{b}}(\mathrm{g})$ & Ratio of yield $^{\mathrm{c}}(\%)$ \\
\hline Gypsum (G) & 6.0 & 1.6 \\
Scutellaria Root (SR) & 3.0 & 42 \\
Gardenia Fruit (GF) & 1.5 & 32 \\
Anemarrhena Rhizome (AR) & 3.0 & 37 \\
Loquat Leaf (LL) & 1.0 & 19 \\
Ophiopogon Tuber (OT) & 6.0 & 36 \\
Lilium Bulb (LB) & 3.0 & 20 \\
Magnolia Flower (MF) & 3.0 & 14 \\
Cimicifuga Rhizome (CR) & 1.5 & 18 \\
Shin'iseihaito (SSHT) & 28 & 23 \\
\hline
\end{tabular}

a All crude drugs are registered in Japanese Pharmacopoeia 17th Edition ${ }^{19}$

${ }^{b}$ Weight of each herbal medicine in a dairy dosage of SSHT. This formula is described in the guideline of over-the-counter Kampo medicine published from Ministry of Health, Labour and Welfare, Japan, 2012.

'The decoctions were prepared according to experimental design, and ratio of yield means \% of dried weight of the decoction to the original crude drug. isolates (A, DDM147; B, DDM179; C, DDM198) from which SSHT extract exhibited the strongest anti-bacterial effects, and investigated the contribution of each crude drug composing of SSHT. SSHT composes G, $\mathrm{SR}, \mathrm{GF}$ and AR as heat-clearing drugs to treat internal heat-syndrome, $\mathrm{MF}$ and CR as exterior-releasing drugs to treat the exterior syndromes of wind-cold and wind-heat, and others. ${ }^{14}$ In Kampo medicine, yellowish discharge from nasal cavities which relates to the bacterial infection is the reflection of lung-heat and the infection of exterior wind-heat pathogen. Then, we considered these drug groups might contribute the anti-MRSA effects of SSHT. We prepared the extracts of SSHT without heat-clearing drugs (SSHT-HC) or exterior-releasing drugs (SSHT-ER) to evaluate the contribution of these groups to the effect of SSHT. As shown in Figure 1, the antibacterial activities of SSHT-HC $(3.0 \mathrm{mg} /$ disk $)$ and SSHT-ER extract $(6.5 \mathrm{mg} /$ disk) against $3 \mathrm{MRSA}$ clinical isolates were significantly decreased compared with SSHT extract $(7.5 \mathrm{mg} / \mathrm{disk})$, and the antibacterial activity of SSHT-HC extract was decreased to control level. The results suggest that heat-clearing drugs mainly contribute the anti-MRSA activity of SSHT, and exterior-releasing drugs partially contribute it.

We investigated anti-MRSA activity of the extract of SSHT without each crude drug consisting of SSHT. As shown in Figure 2, Anti-MRSA activities of the extract of SSHT without G, GF, AR, OT, LB, or MF were equal compared with SSHT extract, however, those of the extract of SSHT without LL were significantly decreased in 1 strain, those of the extract of SSHT without CR were significantly decreased in 2 strains, and those of the extract of SSHT without SR were significantly decreased in 3 strains compared with SSHT extract, and the anti-MRSA activity of SSHT without SR was disappeared to the control level. These results suggest that SR mainly, and LL and CR slightly, contributes anti-MRSA activity of SSHT against MRSA. The diameter of inhibitory zone of SSHT-CR extract in 3 MRSA clinical isolates were decreased $0.6-1.0 \mathrm{~mm}$ and SSHT-MF extract in them were equal compared with SSHT extract. In contrast, the diameter of inhibitory zone of SSHT-ER extract in them were decreased $1.3-2.0 \mathrm{~mm}$. It is considered that SR is the representatives of heat-clearing drugs and the presence of a synergistic or additional effects between CR and MF in exterior-releasing drugs in SSHT.

We screened the antibacterial activity of the hot water extracts of each crude drug composing SSHT. As shown in Figure 3, hot water extracts

\section{Table 2: Weight and ratio of yield of each modified SSHT prescription.}

\begin{tabular}{ccc}
\hline Modified SSHT & $\begin{array}{c}\text { Weight } \\
(\mathbf{g})\end{array}$ & $\begin{array}{c}\text { Ratio of } \\
\text { yield }^{\text {b }} \text { (\%) }\end{array}$ \\
\hline SSHT without ${ }^{\text {d }}$ heat-clearing drugs (SSHT-HC) & 14.5 & 18 \\
SSHT without ${ }^{\text {e }}$ exterior-releasing drugs (SSHT-ER) & 23.5 & 24 \\
SSHT without G (SSHT-G) & 22.0 & 25 \\
SSHT without SR (SSHT-SR) & 25.0 & 19 \\
SSHT without GF (SSHT-GF) & 26.5 & 21 \\
SSHT without AR (SSHT-AR) & 25.0 & 22 \\
SSHT without LL (SSHT-LL) & 27.0 & 20 \\
SSHT without OT (SSHT-OT) & 22.0 & 22 \\
SSHT without LB (SSHT-LB) & 25.0 & 21 \\
SSHT without MF (SSHT-MF) & 25.0 & 23 \\
SSHT without CR (SSHT-CR) & 26.5 & 23 \\
\hline
\end{tabular}

${ }^{a}$ Weight of each herbal medicine related to the dairy dosage of SSHT.

'The decoctions were prepared according to experimental design, and ratio of yield means \% of dried weight of the decoction to the original crude drug.

${ }^{c}$ Heat-clearing drugs refers to G, SR, GF and AR.

${ }^{\mathrm{d}}$ Exterior-releasing drug refers to MF and CR. 

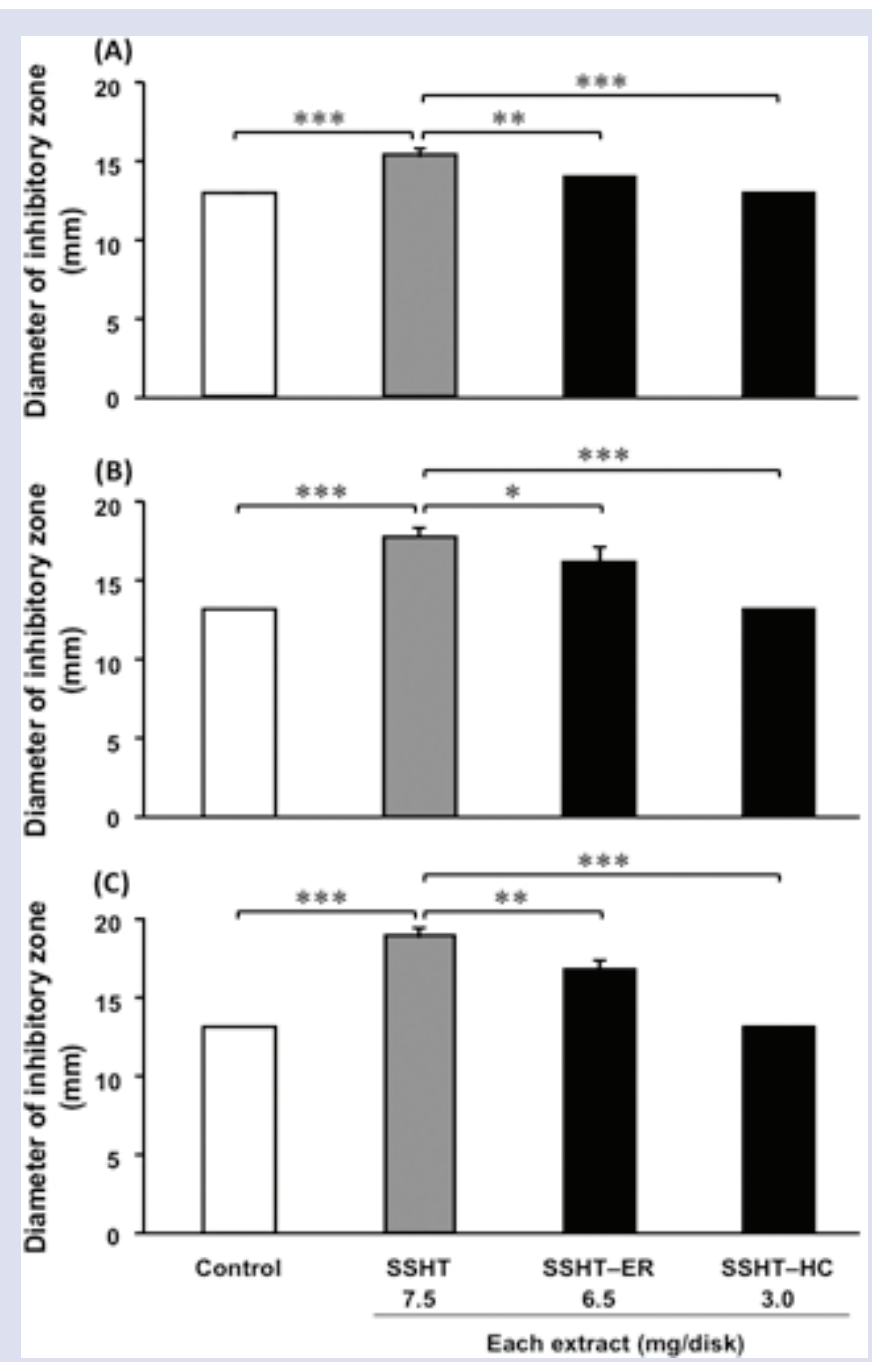

Figure 1 : Antibacterial effect of the extract of SSHT, SSHT-HC and SSHTER against 3 MRSA clinical isolates.

Paper disks containing SSHT, SSHT-HC, or SSHT-ER extract were put on placed on the plates inoculated with each MRSA clinical isolate (A, DDM147; B, DDM179; C, DDM198), and the diameter of inhibitory zone after $18-24 \mathrm{~h}$ incubation was measured. The amount of SSHT-HC and SSHT-ER was equivalent to SSHT extract ( $7.5 \mathrm{mg} /$ disk). Values are expressed as the mean \pm S.D. $(n=3) .{ }^{*} P<0.05,{ }^{* *} P<0.01$, ${ }^{* * *} P<0.001$ evaluated by Bonferroni/Dunnet's multiple $t$-test.

of SR, CR, MF and LL showed significant anti-MRSA clinical isolates, and hot water extracts of SR exhibited the strongest antibacterial activity among 3 strains.

In the theory of traditional Chinese medicine described in the textbook, ${ }^{14}$ SR clears heat and dries dampness, resolves toxicity in the upper burner, and calms the fetus, LL transforms phlegm and clears lung's heat, CR discharges exterior condition, and resolves toxicity in the upper, and MF expels wind-cold and unblocks the nasal passages. As regards other crude drugs composing SSHT, OT, GF, AR, and G also clear heat in the traditional theory. ${ }^{14}$ Therefore, we consider that the term heat in traditional theory and bacterial infection are not equal because not all crude drugs that have the effectiveness of clearing heat exhibit antibacterial activity. In the present study, we showed the hot water extract of SSHT had the antibacterial activity against MRSA. We previously reported that the hot water extract of SR had the antibacterial activity against Streptococcus

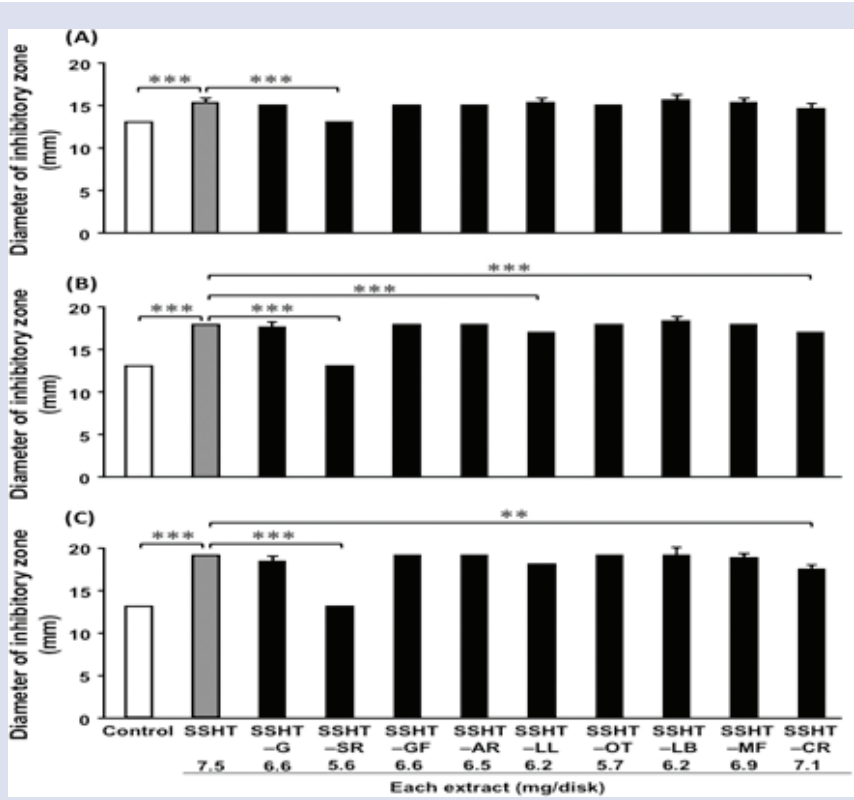

Figure 2 : Antibacterial effects of the extract of SSHT without single crude drug consisting of SSHT against 3 MRSA clinical isolates. Paper disks containing SSHT extract or SSHT-G, SSHT-SR, SSHT-GF, SSHT-AR, SSHT-LL, SSHT-OT, SSHT-LB, SSHT-MF and SSHT-CR were put on placed on the plates inoculated with each MRSA clinical isolate (A, DDM147; B, DDM179; C, DDM198), and the diameter of inhibitory zone after $18-24 \mathrm{~h}$ incubation was measured. Values are expressed as the mean \pm S.D. $(n=3) .{ }^{* *} P<0.01,{ }^{* * *} P<0.001$ evaluated by Bonferroni/ Dunnet's multiple $t$-test.

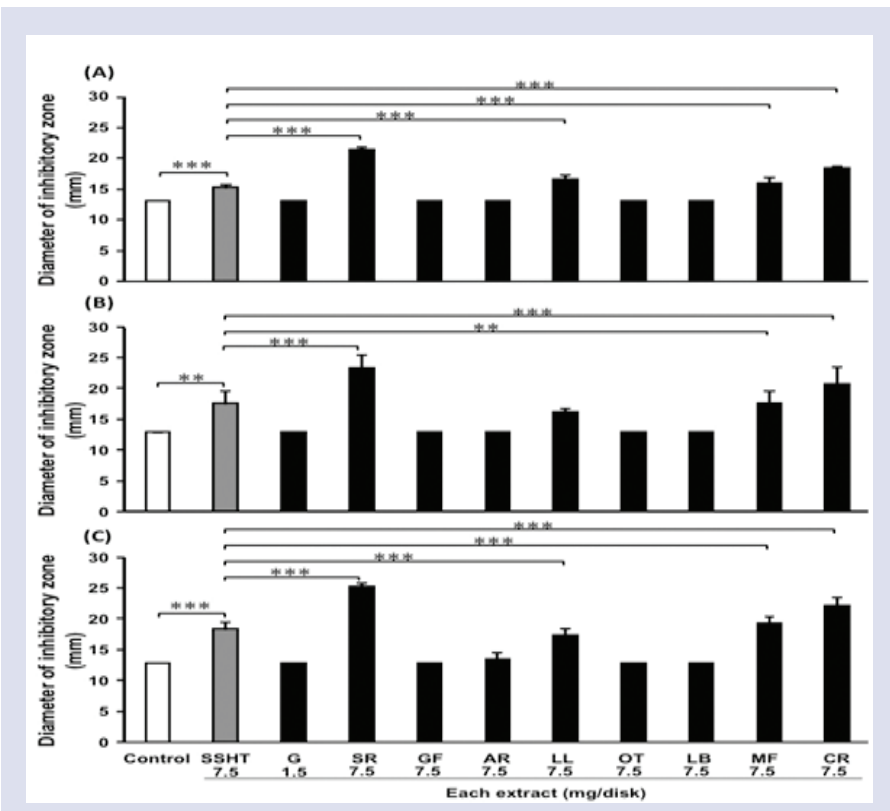

Figure 3 : Antibacterial effects of SSHT extract and the extracts of 9 crude drugs consisting of SSHT against 3 MRSA clinical isolates. Paper disks containing SSHT extract or each extract of G, SR, GF, AR, LL, $\mathrm{OT}, \mathrm{LB}, \mathrm{MF}$ and $\mathrm{C}$ were put on placed on the plates inoculated with each MRSA clinical isolate (A, DDM147; B, DDM179; C, DDM198), and the diameter of inhibitory zone after $18-24 \mathrm{~h}$ incubation was measured. Values are expressed as the mean \pm S.D. $(n=3) .{ }^{* *} P<0.01,{ }^{* * *} P<0.001$ evaluated by Bonferroni/Dunnet's multiple $t$-test. 
pneumoniae. ${ }^{13}$ In addition, it is reported that the hot water extract of SR had the antibacterial activity against Propionibacterium acnes ${ }^{15}$ and Helicobacter pylori, ${ }^{16}$ and $60 \%$ ethanol extract of SR had antibacterial activity against Staphylococcus aureus, ${ }^{17}$ Listeria monocytogenes, and Salmonella enterica serovars Kentucky, Senftenberg, Enteritidis and Typhimurium. ${ }^{18}$ P. acnes and Staphylococcus aureus belong to grampositive bacteria, but $H$. pylori and Salmonella enterica are gram-negative. It is suggested that SR water extract would have broad spectrum antibacterial activity. Bacterial sinusitis is frequently caused by Streptococcus pneumoniae, Haemophilus influenzae, Moraxella catarrhalis, and Streptococcus pyogenes other than MRSA. These bacteria are also gram-positive or gram-negative bacteria. Therefore, SSHT may exhibit antimicrobial activity in these causative bacteria of sinusitis.

We previously measured the amount of baicalin in SR extract using HPLC because baicalin is the marker compounds of $\mathrm{SR}^{13}$, and the content of baicalin in SR extract was 33 (w/w) \%. This means $7.5 \mathrm{mg}$ of SR extract contains $2.5 \mathrm{mg}$ of baicalin. Baicalin has the antibacterial activities against Streptococcus pneumonia, ${ }^{13}$ Sarcina lutea, Staphylococcus aureus, Listeria monocytogenes, Salmonella enterica serovars Kentucky, Senftenberg, Enteritidis and Typhimurium. ${ }^{18}$ It is considered that baicalin may active component of SSHT extract against MRSA.

\section{CONCLUSION}

The hot water extract of SSHT, a formula in traditional Japanese Kampo medicine and Chinese medicine, showed the antibacterial activity against MRSA, and among the components of SSHT, SR mainly contributes antibacterial activity of SSHT against MRSA. Although SSHT contains other crude drugs than SR that have anti-MRSA activity and these crude drugs might exhibit synergistic activity, this effect of crude drugs might be related to one of the action mechanisms of SSHT to treat bacterial sinusitis.

\section{ACKNOWLEDGEMENT}

Nil

\section{CONFLICT OF INTEREST}

\section{None}

\section{ABBREVIATIONUSED}

ANOVA: A one-way analysis of variance; AR: Anemarrhena Rhizome; CR: Cimicifuga Rhizome; GF: Gardenia Fruit; G: Gypsum; LB: Lilium Bulb; LL: Loquat Leaf; MF: Magnolia Flower; OT: Ophiopogon Tuber; SR: Scutellaria Root; SSHT: Shin'iseihaito (xinyiqingfeitang in Chinese).

\section{REFERENCES}

1. Metson RB, Gliklich RE. Clinical outcomes in patients with chronic sinusitis. Laryngoscope. 2000;110(S94):24-8. https://doi.org/10.1097/00005537-20000300200007; PMid:10718411.
2. Birch DS, Saleh HA, Wodehouse T, Simpson IN, Mackay IS. Assessing the quality of life for patients with chronic rhinosinusitis using the "Rhinosinusitis Disability Index". Rhinology. 2001;39(4):191-6. PMid:11826687.

3. Rudmik L, Smith TL. Quality of life in patients with chronic rhinosinusitis. Curr Allergy Asthma Rep. 2011;11(3):247-52. https://doi.org/10.1007/s11882-0100175-2; PMid:21234819.

4. Brook I. Microbiology of sinusitis. Proc Am Thorac Soc. 2011;8(1):90-100. https://doi.org/10.1513/pats.201006-038RN; PMid:21364226.

5. Manarey CR, Anand VK, Huang C. Incidence of methicillin-resistant Staphylococcus aureus causing chronic rhinosinusitis. Laryngoscope. 2004;114(5):939-41. https://doi.org/10.1097/00005537-200405000-00029; PMid:15126760.

6. Huang WH, Hung PK. Methicillin-resistant Staphylococcus aureus infections in acuterhinosinusitis. Laryngoscope. 2006;116(2):288-91. https://doi.org/10.1097/ 01.mlg.0000197316.36698.c4; PMid:16467721.

7. Brook I, Foote PA, Hausfeld JN. Increase in the frequency of recovery of methicillin-resistant Staphylococcus aureus in acute and chronic maxillary sinusitis. J Med Microbiol. 2008;57(8):1015-7. https://doi.org/10.1099/jmm.0.2008/0008510; PMid:18628504.

8. Chu DT, Plattner JJ, Katz L. New directions in antibacterial research. J Med Chem. 1996;39(20):3853-74. https://doi.org/10.1021/jm960294s; PMid:8831751.

9. Limbago BM, Kallen AJ, Zhu W, Eggers P, McDougal LK, Albrecht VS. Report of the $13^{\text {th }}$ vancomycin-resistant Staphylococcus aureus isolate from the United States. J Clin Microbiol. 2014;52(3):998-1002. https://doi.org/10.1128/ JCM.02187-13; PMid:24371243 PMCid:PMC3957794.

10. Melo-Cristino J, Resina C, Manuel V, Lito L, Ramirez M. First case of infection with vancomycin-resistant Staphylococcus aureus in Europe. Lancet. 2013; 382(9888):205. https://doi.org/10.1016/S0140-6736(13)61219-2.

11. The Japan Society for Oriental Medicine. Introduction to Kampo. Tokyo, Japan: Elsevier Japan KK;2005

12. Minami M, Konishi T, Jiang Z, Arai T, Makino T. Effect of shin'iseihaito on lung colonization of pneumococcus in murine model. African Journal Trade Company Alternate Med. 2015;12(6):131-9. https://doi.org/10.4314/ajtcam.v12i6.13.

13. Konishi T, Minami M, Jiang Z, Arai T, Makino T. Antibacterial activity of Shin'iseihaito (Xin Yi Qing Fei Tang) against Streptococcus pneumoniae. Pharmacogn J. 2016;8(1):20-3. https://doi.org/10.5530/pj.2016.1.4.

14. Bensky D, Clavey S, Stoger E. Chinese herbal medicine Materia Medica $3^{\text {rd }}$ Ed. Eastland Press, Seatlle, WA, USA.

15. Higaki S, Nakamura M, Morohashi M, Hasegawa Y, Yamagishi T. Activity of eleven kampo formulations and eight kampo crude drugs against Propionibacterium acnes isolated from acne patients: retrospective evaluation in 1990 and 1995. J Dermatol. 1996;23(12):871-5. https://doi.org/10.1111/j.1346-8138.1996.tb02717.x; PMid:9037918.

16. Ma F, Chen Y, Li J, Qing HP, Wang JD, Zhang YL, et al. Screening test for antihelicobacter pylori activity of traditional Chinese herbal medicines. World J Gastroenterol. 2010;16(44):5629-34. https://doi.org/10.3748/wjg.v16.i44.5629; PMid:21105198 PMCid:PMC2992683.

17. Kubo M, Kimura Y, Odani T, Tani T, Namba K. Studies on Scutellariae Radix. Part II: The antibacterial substance. Planta Med. 1981;43(2):194-201. https://doi org/10.1055/s-2007-971499; PMid:7312987.

18. Lu Y, Joerger R, Wu C. Study of the chemical composition and antimicrobial activities of ethanolic extracts from roots of Scutellaria baicalensis Georgi. J Agric Food Chem. 2011;59(20):10934-42. https://doi.org/10.1021/jf202741x; PMid:21866919.

19. The Society of Japanese Pharmacopoeia. Japanese Pharmacopoeia $17^{\text {th }}$ Ed. 2016. Tokyo, Japan: Yakuji-Nippo.

\section{SUMMARY}

- $\quad$ Shin'iseihaito (xin yi qing fei tang in Chinese, SSHT), a formula in traditional Japanese Kampo medicine and Chinese medicine comprising nine crude drugs, showed antibacterial effect against methicillin-resistant Staphylococcus aureus (MRSA)

- $\quad$ Each water extract of Scutellaria Root, Loquat Leaf, Magnolia Flower and Cimicifuga Rhizome showed significant anti-MRSA activity, and Scutellaria Root extract exhibited the largest inhibitory zone. 


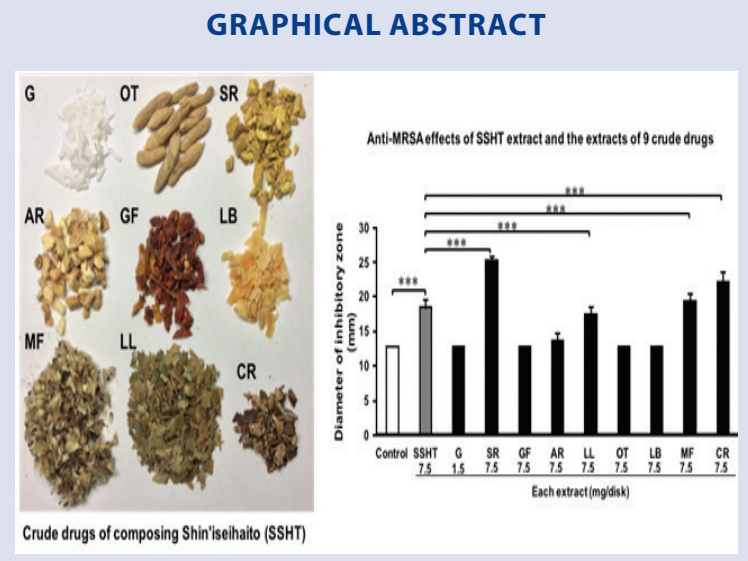

\section{ABOUT AUTHOR}

Toru Konishi: He is a Ph. D. student at Department of Pharmacognosy, Graduate School of Pharmaceutical Sciences, Nagoya City University. He graduated in Bachelor of Faculty of Pharmaceutical Sciences, Hokkaido University, and got a license of Pharmacist in Japan. His doctoral research focused on the evaluation of anti-rhinitis activity of Japanese traditional Kampo medicine.

Cite this article: Konishi T, Minami M, Makino T. Antibacterial Activity Of Shin'iseihaito (Xinyiqingfeitang) And its Components Against Methicillin-Resistant Staphylococcus aureus. Pharmacogn J. 2017;9(3):310-4. 